\title{
Analysis of futsal matches using a single-camera computer vision system
}

\author{
Heloiza M Paulichen*, Kallil M C Zielinski ${ }^{\dagger}$, Dalcimar Casanova ${ }^{\ddagger}$ and Pablo G Cavalcanti ${ }^{\S}$ \\ Universidade Tecnológica Federal do Paraná \\ Pato Branco, Paraná, Brasil \\ Email: *paulichen@alunos.utfpr.edu.br, ${ }^{\dagger}$ kallil@alunos.utfpr.edu.br, ${ }^{\ddagger}$ dalcimar@utfpr.edu.br, ${ }^{\S}$ pgcavalcanti@utfpr.edu.br
}

\begin{abstract}
Resumo-The use of computer systems in sports has increased significantly in the last decade. Consequently, systems have been developed to help each athlete or team quantify their performance, such as distances traveled, speeds attained, and positions where each athlete was on the court or field. In this work, a method based on computer vision is proposed to analyse futsal matches. Videos were acquired using a single camera with a wide-angle lens, which facilitates the installation and calibration process in different matches and arenas. The approach is illustrated through video recordings of Pato Futsal team, from which the athletes were detected, their positions projected from pixels to real world coordinates and their trajectories estimated. The generated data visualization aims to help coaches in their physical and tactical analysis.
\end{abstract}

\section{Introdução}

Assim como o futebol, o futsal vem conquistando seu espaço no cenário mundial e no Brasil [1]. Esse crescimento recente tem levado a um aumento da demanda por informações estatísticas relacionadas as partidas e aos seus jogadores de forma individual e coletiva. Algumas dessas estatísticas são tradicionalmente anotadas manualmente pelos mesários, como tempo de bola rolando, tempo de cada jogador em quadra, etc. Por outro lado existe uma série de dados que são muito complicados para se obter de forma manual, tal como a distância percorrida por cada jogador, a região da quadra de mais atuação e até as iterações entre os jogadores (e.g. roubadas de bola, marcação, etc). O estudo dessas interações entre os atletas é de especial importância em esportes coletivos, como futebol e futsal [2].

Esse tipo de informação estatística mais detalhada é importante subsídio para elevar o desempenho geral da equipe, pois possibilita à equipe técnica evidenciar o padrão tático utilizado por seus adversários assim como planejar o treinamento físico e tático de seus atletas [3]. Permite também que o público geral compreenda melhor esse esporte em suas qualidades e complexidades, melhorando a qualidade das transmissões e análises pós-jogo [4].

Todavia, a aquisição desse tipo de dado demanda um sistema automatizado, que pode ser concebido por uma série de sensores ou através da aquisição de imagens. A abordagem por imagens é, sob um determinado ponto de vista, menos complexa, pois dispensa que cada jogador tenha de carregar um dispositivo e também dispensa a instalação de uma série de antenas para captura dos sinais. A tecnologia de medição baseada na utilização de câmeras e vídeos é conhecida como videogrametria [5] e pode ser combinada com outras técnicas de visão computacional.

Entretanto, a abordagem por visão computacional no futsal ainda é considerada escassa em estudos científicos [4]. Nesta linha, avanços recentes dos métodos têm motivado um aumento no interesse de tal abordagem.

Neste trabalho, um método foi desenvolvido para segmentação e, posterior análise estatística, dos jogadores de linha de uma equipe de Futsal. Tanto para o treinamento quanto para avaliação do método, foram utilizados vídeos de partidas da equipe Pato Futsal, gravados no ginásio Dolivar Lavarda em Pato Branco - PR. Em uma análise inicial, foi medida a distância percorrida de cada jogador da equipe Pato Futsal e o respectivo mapa de calor gerado pela posição do jogador na quadra ao longo do tempo da partida.

Adicionalmente, optou-se por utilizar uma única câmera de lente grande angular para captura dos vídeos. Essa abordagem facilita a aquisição da imagem, uma vez que quase toda a quadra está dentro do campo de visão do dispositivo único de captura, algo que não seria possível com lentes convencionais, dado o espaço disponível dentro da maioria dos ginásios de futsal.

\section{Trabalhos relacionados}

Outros trabalhos já abordaram a análise de partidas de futsal por meio de processamento de imagens e visão computacional, e nesta seção eles são apresentados.

Em [6], o autor utilizou três câmeras para obter as gravações de uma partida de futsal. Após a obtenção dos vídeos, foi necessário realizar uma sincronização para que fossem processados quadros em um mesmo instante de tempo. A calibração foi realizada manualmente, dependendo de um usuário para reconhecer pontos comuns aos dois planos e marcá-los como equivalentes. A partir da calibração, matrizes homográficas foram geradas para calcular a correspondência entre os planos. Finalmente, para a detecção dos jogadores, um Modelo de Mistura de Gaussianas (MMG) foi utilizado para segmentação, que busca modelar o fundo a partir de um conjunto de imagens passadas.

Outra abordagem por [7] também utilizou um MMG para a remoção de fundo, mas com apenas uma câmera 
estática posicionada no topo da quadra. O objetivo era analisar a movimentação dos jogadores de futsal usando um filtro de particulas preditivo, que usa informações do estado atual de um objeto para inferir seu estado no próximo instante de tempo. Os resultados das movimentações foram mostrados em gráficos onde o plano de fundo era a própria quadra de futsal e a trajetória dos jogadores era destacada por linhas.

Uma análise sobre a correção de distorção radial em câmeras de lente grande angular no rastreamento de jogadores de futsal é feita por [8]. Quatro câmeras foram usadas para gravar uma partida das quartas de final da Liga de Futsal de São Paulo em 2013, três câmeras possuíam lentes convencionais e uma câmera era de lente grande angular. Para corrigir a distorção radial, foi aplicada a transformação de Hough para identificar linhas distorcidas nas imagens. A calibração de câmera foi feita baseada em 23 pontos de controle em uma superfície de corte, com as distâncias reais previamente medidas. A trajetória dos jogadores foi obtida pelo software DVideo. Uma comparação entre os resultados para as câmeras de lentes convencionais e a câmera de lente grande angular é mostrada e os resultados indicam que o uso de uma câmera de lente grande angular com correção de distorção radial é similar às câmeras de lente convencionais, mas com a vantagem de que o campo de visão é maior, tornando possível a captura da quadra inteira.

\section{Referencial Teórico}

\subsection{Regras do Futsal}

O futsal é uma modalidade esportiva coletiva, em que as disputas ocorrem entre equipes com cinco integrantes de cada lado. A quadra deve ser retangular e as dimensões oficiais para partidas nacionais são de, no mínimo, 25m x $16 \mathrm{~m}$ e, no máximo $42 \mathrm{~m} \times 25 \mathrm{~m}$. Já para partidas internacionais, as dimensões são de, no mínimo, $38 \mathrm{~m}$ x $20 \mathrm{~m}$ e, no máximo, $42 \mathrm{~m} \times 25 \mathrm{~m}[9]$.

\subsection{Detecção de jogadores}

Para a detecção dos jogadores na quadra, o método baseado em rede neural YOLOv3 (You Only Look Once) foi escolhido. Esse modelo de rede apresenta um sistema de detecção de objetos em tempo real que vem apresentando bons resultados na literatura. Em [10], a rede YOLOv3 apresentou uma precisão média de detecções (mAP) de $57,9 \%$ no conjunto de dados MS COCO (Microsoft Common Objects in COntext, processando a 30 quadros por segundo.

Para a extração de características, essa rede usa camadas convolucionais consecutivas de $3 \times 3$ e $1 \times 1$, possuindo no total 53 camadas convolucionais. Essa estrutura é denominada de Darknet-53 e é apresentada na Tabela 1.

$\mathrm{Na}$ arquitetura Darknet-53, cada camada convolucional é seguida de uma camada de normalização em lote e pela ativação do Leaky ReLU. Nenhuma forma de pooling é usada e uma camada convolucional com stride 2 é usada

\begin{tabular}{|c|c|c|c|}
\hline Tipo & Filtros & Tamanho & Saída \\
\hline Convolucional & 32 & $3 \times 3$ & $256 \times 256$ \\
\hline Convolucional & 64 & $3 \times 3 / 2$ & $128 \times 128$ \\
\hline Convolucional & 32 & $1 \times 1$ & \\
\hline Convolucional & 64 & $3 \times 3$ & \\
\hline Residual & & & $128 \times 128$ \\
\hline Convolucional & 128 & $3 \times 3 / 2$ & $32 \times 32$ \\
\hline Convolucional & 64 & $1 \times 1$ & \\
\hline Convolucional & 128 & $3 \times 3$ & \\
\hline Residual & & & $64 \times 64$ \\
\hline Convolucional & 256 & $3 \times 3 / 2$ & $32 \times 32$ \\
\hline Convolucional & 128 & $1 \times 1$ & \\
\hline Convolucional & 256 & $3 \times 3$ & \\
\hline Residual & & & $32 \times 32$ \\
\hline Convolucional & 512 & $3 \times 3 / 2$ & $16 \times 16$ \\
\hline Convolucional & 256 & $1 \times 1$ & \\
\hline Convolucional & 512 & $3 \times 3$ & \\
\hline Residual & & & $16 \times 16$ \\
\hline Convolucional & 1024 & $3 \times 3 / 2$ & $8 \times 8$ \\
\hline Convolucional & 512 & $1 \times 1$ & \\
\hline Convolucional & 1024 & $3 \times 3$ & \\
\hline Residual & & & $8 \times 8$ \\
\hline Média de Pooling & & Global & \\
\hline Densa & & 1000 & \\
\hline Softmax & & & \\
\hline
\end{tabular}

Tabela 1: Estrutura do modelo Darknet-53.

Adaptado de: [10].

para reduzir a amostragem dos mapas de características. Isso ajuda a impedir a perda de características de baixo nível geralmente atribuídos ao pooling. Para uma imagem de entrada com dimensão 416 x 416, é feita uma grade de 13 x 13 células. Cada célula da grade deve ser responsável por encontrar o local exato e a categoria à qual o objeto pertence. O Darknet-53 usa três escalas para detectar objetos grandes, objetos médios e objetos pequenos. Os três tamanhos relativos dos mapas de características resultantes são 13 x 13, 26 x 26 e 52 x 52 [11].

\subsection{Remoção de Fundo}

Para que a detecção de jogadores seja mais eficiente, um pré-processamento de remoção de fundo foi realizado nas imagens do vídeo. Este pré-processamento tem por objetivo retirar uma grande quantidade de objetos estáticos e semiestáticos que não são de interesse da análise proposta (e.g. as traves, as linhas da quadra, publicidades, etc).

De acordo com [12], uma das abordagens mais simples para a detecção de mudanças entre dois quadros de imagem $f\left(x, y, t_{i}\right)$ e $f\left(x, y, t_{j}\right)$, tomados nos momentos $t_{i}$ e $t_{j}$, respectivamente, é comparar as duas imagens pixel por pixel. Uma forma de fazer isso é criar uma imagem que represente a diferença entre a imagem referência e outra imagem subsequente na mesma cena, em que a imagem referência contém apenas componentes estáticos. Ao compará-las, a imagem diferença elimina os elementos fixos em ambas as imagens e mostra os componentes em movimento. Mais detalhes sobre a imagem de referência utilizada neste estudo são apresentados na Seção 4. 


\subsection{Correção de lentes e transformação projetiva}

Como neste trabalho optou-se por utilizar aquisição dos vídeos com um dispositivo de lente grande angular (popularmente conhecidas como "olho de peixe"), é necessária uma correção da distorção gerada por essas lentes. Esta correção é responsável por determinar a posição de cada pixel em um espaço não distorcido a partir da imagem original, obtendo assim uma imagem retilínea [13].

Após a correção da distorção, é necessário aplicar transformações projetivas para realizar o mapeamento dos pontos da dimensão $3 \mathrm{D}$ da quadra $(x, y, z)$ em pixels da imagem 2D $\left(x^{\prime}, y^{\prime}\right)$. Possibilitando realizar medições em cada quadro dos vídeos das partidas.

Segundo [14], dados dois espaços projetivos de dimensões $m$ e $n$, as transformações $T: \mathbb{R P}^{m} \longrightarrow \mathbb{R P}^{n}$ são dadas por transformações $T: \mathbb{R}^{m+1} \longrightarrow \mathbb{R}^{n+1}$, chamadas transformações projetivas.

Para este trabalho, foi utilizada a homografia, que é uma transformação projetiva planar, ou seja, mapeia pontos de um plano $\pi$ para outro plano $\pi^{\prime}$, como mostra a Figura 1 . Em aspectos práticos, um plano da homografia é representado pelos quadros dos vídeos e o plano resultante definido foi a vista superior da quadra.

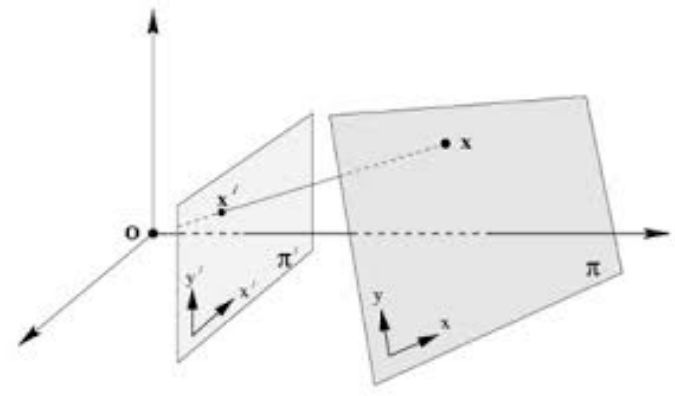

Figura 1: Mapeamento entre planos utilizando homografia. Fonte: [15]

\subsection{Rastreamento e otimização da trajetória}

Conforme descrito anteriormente, os jogadores são detectados em cada quadro do vídeo. Para que eles sejam associados entre um quadro e outro, ou seja, para rastrear os jogadores, uma posição no centro inferior de cada bounding box foi estimada, representando os pés dos jogadores. Foi estabelecido um ponto inicial $\left(x_{0}, y_{0}\right)$ onde um jogador foi detectado em um determinado quadro e, então, houve uma comparação com o quadro seguinte. $\mathrm{O}$ jogador detectado em uma posição $\left(x_{1}, y_{1}\right)$, que estava mais próximo à posição inicial $\left(x_{0}, y_{0}\right)$, foi considerado o mesmo atleta do quadro anterior.

Entretanto, este processo de rastreamento pode acarretar em erros, seja por oclusão de jogadores em determinados quadros ou mesmo por imprecisões do algoritmo de detecção. Para minimizar esse efeito, foi utilizado o filtro de Kalman.
O filtro de Kalman possui inúmeras aplicações, sendo comum para orientação, navegação e controle de veículos, tais como aeronaves e navios [16]. Além disso, o filtro de Kalman tem seu conceito amplamente difundido em análises de série temporais usadas no campo de processamento de sinais. Os filtros Kalman também são um dos principais tópicos no controle de movimento robótico, e às vezes são incluídos na otimização de trajetória [17].

$\mathrm{O}$ filtro de Kalman assume que o estado real em um tempo $k$ é proveniente de um estado em $(k-1)$ de acordo com:

$$
x_{k}=F_{k} x_{k-1}+B_{k} u_{k}+w_{k}
$$

em que $F_{k}$ é o modelo de transição de estado aplicado ao estado anterior em $x_{k-1}, B_{k}$ é o modelo de controle de entrada aplicado ao vetor $u_{k}$, e $w_{k}$ é o ruído do processo, o qual se supõe ser extraído de uma distribuição normal multivariada de média zero, $\mathcal{N}$, com covariância $Q_{k}: w_{k} \sim$ $\mathcal{N}\left(0, Q_{k}\right)$.

Em $k$ uma medida $z_{k}$ do estado real $x_{k}$ é feita de acordo com:

$$
z_{k}=H_{k} x_{k}+v_{k}
$$

em que $H_{k}$ é o modelo de observação que mapeia o espaço de estado real no espaço observado e $v_{k}$ é o ruído de observação que é assumido como sendo um ruído branco gaussiano de média zero com covariância $R_{k}$ : $v_{k} \sim \mathcal{N}\left(0, R_{k}\right)$.

O estado inicial e os vetores de ruído a cada passo $\left\{x_{0}, w_{1}, \ldots, w_{k}, v_{1} \ldots v_{k}\right\}$ são assumidos como sendo mutuamente independentes.

\section{Materiais e método proposto}

A câmera utilizada para este projeto é a GoPro HERO 4 Black Edition. Os vídeos foram gravados com 30 quadros por segundo (fps) e resolução de 1080p SuperView - esta opção utiliza o formato grande angular de lentes, permitindo um campo de visão maior e, por consequência, quase que a totalidade da quadra é visível nas imagens (ver Figura 2). Apesar das redes de proteção da quadra estarem visíveis em partes da imagem, elas não interferiram nos resultados obtidos.

Os testes para o método proposto foram realizados a partir de dois vídeos, sendo cada um de 40 segundos e totalizando 1200 quadros. Aleatoriamente, 50 quadros foram separados para o treinamento da rede YOLOv3 e os demais utilizados para testes.

Inicialmente foi gerada uma imagem referência utilizando a mediana dos canais RGB de 500 quadros de um video controle. A Figura 3 a apresenta esta imagem, a qual foi utilizada como referência para remoção de fundo. As imagens utilizadas no processo de detecção dos jogadores são, portanto, resultados da subtração desta imagem referência a partir das imagens originais. 


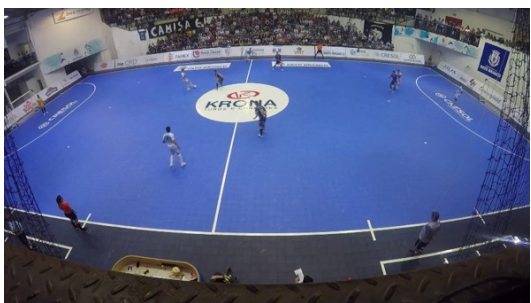

Figura 2: Posicionamento da câmera durante as partidas.

Para o treinamento da rede YOLOv3, foram marcados 4 diferentes bounding boxes em cada quadro de teste, identificando apenas os jogadores de linha, pois a movimentação do goleiro foi ignorada em nossos experimentos. Portanto, 200 bounding boxes referentes aos jogadores da equipe em estudo são utilizados. A partir dos pesos do modelo Darknet53 pré-treinados para o conjunto de dados MS COCO, após 100 épocas, a rede se mostrou capaz de detectar objetos de uma única classe, aqui denominada pato. A Figura $3 \mathrm{~b}$ apresenta um resultado da aplicação do YOLOv3 após este treinamento. É possível notar os 4 bounding boxes correspondentes às posições dos 4 jogadores da equipe em estudo durante o quadro em questão.

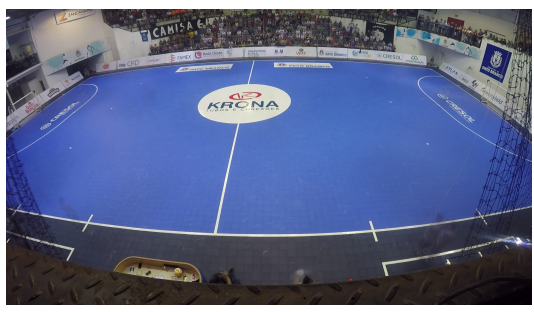

(a) Fundo

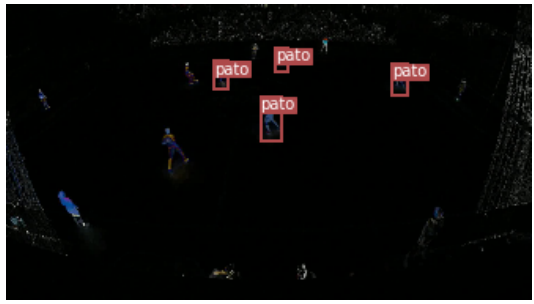

(b) Detecção dos jogadores sem o fundo

Figura 3: Detecção dos jogadores com remoção de fundo.

Identificado os objetos de interesse, a correção da distorção da lente foi realizada utilizando o método proposto em [8], o qual é capaz de corrigir as distorções a partir da identificação automática das linhas da quadra a partir da transformada de Hough [18].

Em sequência, para a transformação projetiva, foi definido um plano com dimensões 400 x 200 pixels, o qual representa a vista superior da quadra. Esta dimensão foi escolhida para estabilizar uma escala de 10:1 com a quadra, cujas dimensões são $40 \mathrm{~m} \times 20 \mathrm{~m}$, tornando mais fácil a conversão de medidas e cálculos como distância percorrida. Aplicando a técnica de homografia na imagem, foi possível obter a representação da vista superior da quadra. Observase que os cantos inferiores desta representação é indefinida, em razão de estarem fora do campo de visão da câmera.

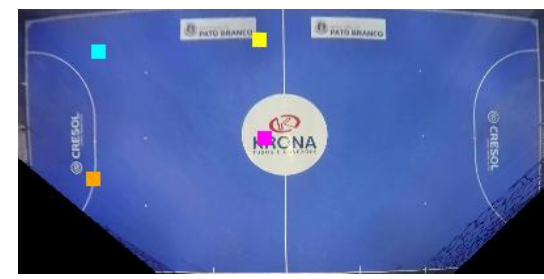

Figura 4: Vista superior da quadra obtida por homografia.

\section{Resultados}

Para ambos os vídeos, foi avaliada a qualidade da detecção dos jogadores dado pelos bounding boxes resultantes da inferência e mensurados a distância percorrida e a posição dos jogadores na quadra. Conforme anteriormente descrito, cada jogador é rastreado quadro-a-quadro e suas trajetórias corrigidas por filtros de Kalman.

Através de mapas de calor (coluna 1 das imagens 6 e 8), apresenta-se visualizações relativas a todas posições de quadra que cada jogador esteve ao longo do vídeo. Onde, cores mais quentes refletem partes da quadra em que o jogador esteve mais tempo presente. Já com gráficos de linha (coluna 2 das imagens 6 e 8), é possível analisar a trajetória completa da movimentação do jogador. Por sua vez, a coluna 3 apresenta uma relação entre distância percorrida ao longo do tempo decorrido.

\subsection{Análise vídeo I}

No primeiro vídeo, ao aplicar a rede YOLOv3 para a detecção dos jogadores, foram detectados quatro bounding boxes para todos os quadros do vídeo, como é possível notar na Figura 5. Pode-se notar que os quatro jogadores do Pato Futsal foram corretamente identificados e estão representados na figura com a cor roxa.

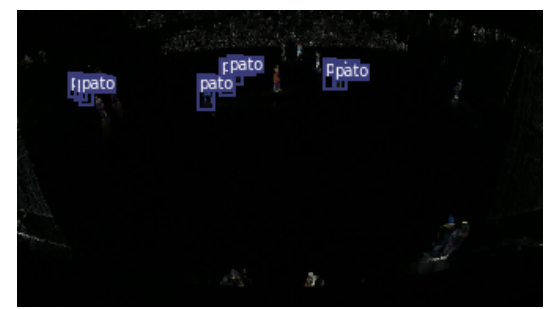

Figura 5: Quadro do Vídeo I sendo analisado pelo YOLOv3.

Após a detecção dos jogadores, os processos de rastreamento, correção de distorção e transformação projetiva, como mencionados na Seção 3 deste trabalho, foram aplicados para, então, coletar os dados sobre as movimentações individuais dos jogadores e apresentá-las como visualizações. O mapa de calor, gráfico de movimentação e distância percorrida pelo tempo são mostrados na Figura 6 . 


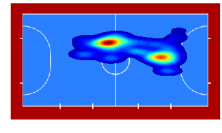

(a)

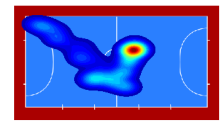

(d)

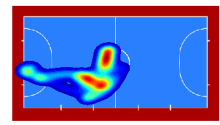

(g)

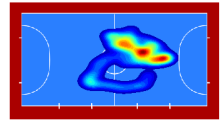

(j)

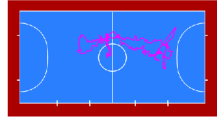

(b)

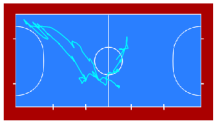

(e)

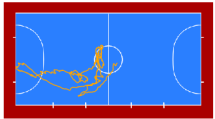

(h)

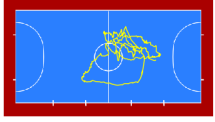

(k)

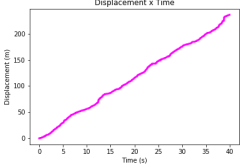

(c)

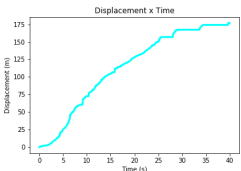

(f)

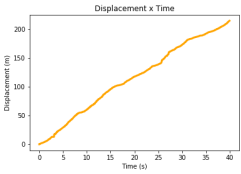

(i)

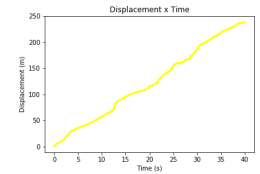

(1)
Figura 6: Visualização de dados para o Vídeo I.

Pode-se observar nessa figura que os jogadores representados pela cor amarela e magenta tiveram suas movimentações mais concentradas na metade direita da quadra (neste momento da partida, respectiva a defesa), enquanto os jogadores representados pelas cores laranja e ciano chegaram algumas vezes próximos ao gol do adversário e suas movimentações se deram na parte esquerda da quadra (região de ataque). Quanto aos gráficos de movimentação, é possível notar que o jogador representado pela cor ciano teve um pico de velocidade dos 5 aos 25 segundos de vídeo, enquanto os demais se movimentaram de forma mais uniforme.

\subsection{Análise vídeo II}

Aplicando o YOLOv3 para o vídeo II, em alguns casos foram detectados mais de quatro bounding boxes, como mostra a Figura 7a. O processo de rastreamento é o responsável por eliminar os sobressalentes, ou seja, aqueles mais distantes das posições dos quatro jogadores no quadro anterior. Neste vídeo, em $94.7 \%$ dos quadros foram detectados 4 ou mais bounding boxes. Porém, também houve casos em que a rede captou menos de quatro bounding boxes, caso representado na Figura 7b. Nestes casos, optamos por repetir a coordenada anterior do jogador que não foi associado pelo processo de rastreamento. Com essas correções, garantiu-se que as coordenadas de cada jogador seriam sempre estimadas e seus movimentos mapeados.

As visualizações geradas para o Vídeo II são apresentadas na Figura 8.

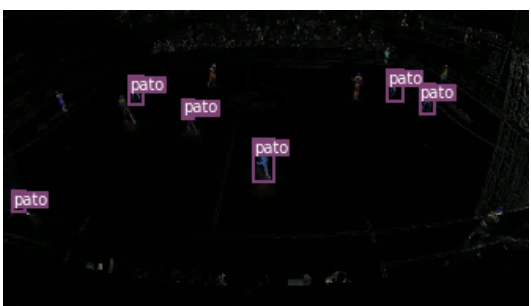

(a) Mais de 4 bounding boxes detectados

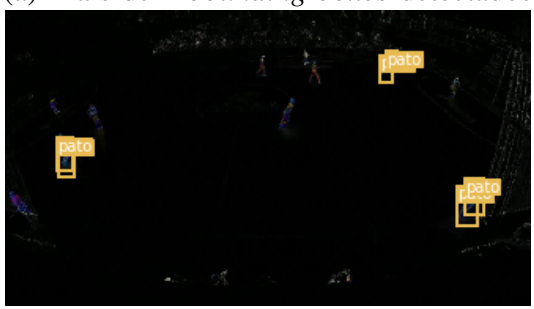

(b) Menos de 4 bounding boxes detectados

Figura 7: Quadros do Vídeo II sendo analisado pelo YOLOv3.

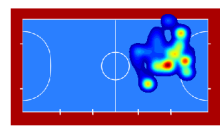

(a)

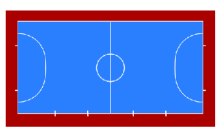

(d)

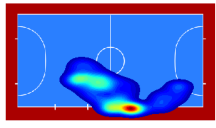

(g)

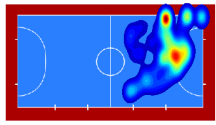

(j)

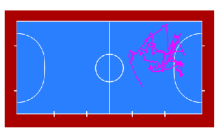

(b)

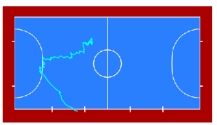

(e)

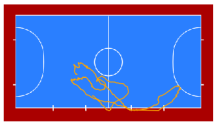

(h)

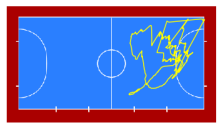

(k)

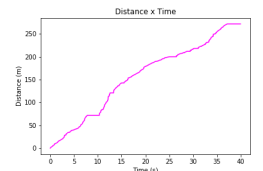

(c)

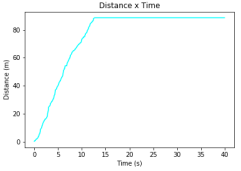

(f)

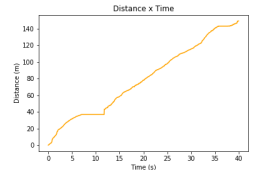

(i)

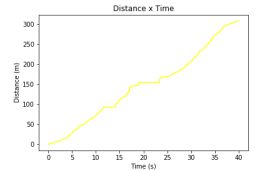

(1)
Figura 8: Visualização de dados para o Vídeo II.

Para esta análise, a detecção do jogador representado pela cor ciano passou a ser trocada pela posição da árbitra, fazendo com que sua referência fosse perdida. Uma possível explicação para isso é que a cor do uniforme da árbitra, após a aplicação da remoção de fundo, ficou similar com a cor do uniforme dos jogadores da equipe Pato Futsal e isso fez com que a detecção se equivocasse. Também é possível identificar na Figura 8 que está acontecendo uma jogada de defesa para a equipe Pato Futsal, pois a movimentação 
dos jogadores se concentrou no campo de defesa. Além disso, em alguns momentos os jogadores ficaram parados em suas posições, como é possível observar nos gráficos de movimentação em função do tempo.

\section{Considerações finais}

Neste artigo, foi proposto um método para análise de partidas de futsal utilizando métodos de visão computacional. Para tal, foram utilizadas etapas de aquisição de imagens, subtração de fundo, detecção de objetos, correção de distorção, transformação projetiva e suavização de erros no processo.

Os resultados obtidos foram satisfatórios, com baixo erro na etapa de detecção de objetos e que foram possíveis de correção. Destaca-se a utilização de câmera única como dispositivo de aquisição, sem necessidade de integração, sincronização ou registro de imagens de diferentes fontes.

A transformação projetiva por homografia é de especial destaque, pois permite que se projete o campo de visão da câmera para uma vista superior da quadra, tornando mais fácil a análise técnica de jogadas e posicionamento do time.

A visualização obtida por mapas de calor permitiu analisar o local da quadra com maior concentração de movimento de cada um dos jogadores individualmente. Essa análise pode gerar subsídios para análise tática do jogo de forma precisa, possivelmente melhorando o desempenho da equipe. Também gera subsídios para avaliar o desempenho físico individual de cada jogador, medido aqui pela distância percorrida em relação ao tempo.

Trabalhos futuros como a identificação do goleiro e dos jogadores adversários, permitindo uma análise mais profunda perante determinados tipos de jogadas dos times adversários, poderão ser realizados a partir deste apresentado.

Com avanço da técnica, é possível criar uma ferramenta para analisar o comportamento e a movimentação dos jogadores durante as partidas, possibilitando consequentemente corrigir os respectivos erros em tempo real.

\section{Referências}

[1] B. H. Soares and H. Tourinho Filho, "Análise da distância e intensidade dos deslocamentos, numa partida de futsal, nas diferentes posições de jogo," Revista Brasileira de Educação Física e Esporte, vol. 20, no. 2, pp. 93-101, 2006.

[2] Z. Niu, X. Gao, and Q. Tian, "Tactic analysis based on real-world ball trajectory in soccer video," Pattern Recognition, vol. 45, no. 5, p. 1937-1947, 2012.

[3] G. Zhu, C. Xu, Y. Rui, S. Jiang, W. Gao, and H. Yao, "Trajectory based event tactics analysis in broadcast sports video," Proceedings of the 15th ACM international conference on Multimedia, vol. 5, pp. 58-67, 2007.

[4] R. Moore, S. Bullough, S. Goldsmith, and L. Edmonson, "A systematic review of futsal literature," American Journal of Sports Science and Medicine, vol. 2, no. 3, pp. 108-116, 2014.

[5] P. J. Figueroa, N. J. Leite, and R. M. Barros, "Tracking soccer players aiming their kinematical motion analysis," Computer Vision and Image Understanding, vol. 101, no. 2, pp. 122-135, 2006.
[6] M. B. de Oliveira, "Detecção Automática de Jogadores de Futsal Baseada em Visão Computacional," 2017, monografia - Curso de Engenharia de Computação, Centro Federal de Educação Tecnológica de Minas Gerais. Belo Horizonte.

[7] P. H. C. d. Pádua, F. L. C. Pádua, and M. T. D. Sousa, "Particle filter-based predictive tracking of futsal players from a single stationary camera," Brazilian Symposium of Computer Graphic and Image Processing, vol. 2015-Octob, no. August, p. 134-141, 2015.

[8] L. H. P. Vieira, E. A. Pagnoca, F. Milioni, R. A. Barbieri, R. P. Menezes, L. Alvarez, L. G. Déniz, D. Santana-Cedrés, and P. R. P. Santiago, "Tracking futsal players with a wide-angle lens camera: accuracy analysis of the radial distortion correction based on an improved hough transform algorithm," Computer Methods in Biomechanics and Biomedical Engineering: Imaging \& Visualization, vol. 5, no. 3, p. 221-231, 2017.

[9] FIFA, Futsal laws of the game 2014/2015. FIFA-Strasse 20, P.O. Box, 8044 Zurich, Switzerland: FIFA, 2014.

[10] J. Redmon and A. Farhadi, "Yolov3: An incremental improvement," arXiv, 2018.

[11] J. Redmon, "Darknet: Open source neural networks in c," 2016. [Online]. Available: http://pjreddie.com/darknet/

[12] R. C. Gonzalez and R. E. Woods, Processamento Digital de Imagens. Av. Ermano Marchetti, 1435 CEP: 05038-001 - São Paulo - SP: Pearson, 2009.

[13] A. C. Valente, "Análise Comparativa de Compressão de Imagens Fisheye, Retilíneas e Panorâmicas," 2010, monografia - Curso de Engenharia Elétrica, Universidade de Brasília, Faculdade de Tecnologia. Brasília.

[14] C. C. dos Santos Cavalcanti Marques, "Um Sistema de Calibração de Câmeras," 2007, dissertação - Mestrado da área de concentração de Computação Gráfica, Universidade Federal de Alagoas, Programa de Pós Graduação em Matemática. Maceió.

[15] M. C. dos Santos, "Revisão de Conceitos em Projeção, Homografia, Calibração de Câmera, Geometria Epipolar, Mapas de Profundidade e Varredura de Planos," Unicamp, Campinas, Tech. Rep., 2012.

[16] P. Zarchan and H. Musoff, Fundamentals of Kalman filtering: a practical approach. American Institute of Aeronautics and Astronautics, Inc., 2013.

[17] R. E. Kalman, "A new approach to linear filtering and prediction problems," Journal of basic Engineering, vol. 82, no. 1, pp. 35-45, 1960.

[18] A. Shehata, S. Mohammad, M. Abdallah, and M. Ragab, "A survey on hough transform, theory, techniques and applications," 022015. 